\title{
THE RADIO LOBES OF VIRGO A
}

\author{
H. ROTTMANN, K.-H. MACK, U. KLEIN \\ Radioastronomisches Institut der Universität Bonn - Auf dem \\ Hügel 71, 53121 Bonn, Germany \\ R. WIELEBINSKI \\ Max-Planck Institut für Radioastronomie - Auf dem Hügel 69, \\ 53121 Bonn, Germany \\ N. KASSIM \\ Naval Research Laboratory, Washington DC 20375-5351, USA \\ AND \\ R. PERLEY \\ NRAO, P.O. Box 0, Socorro, New Mexico 87801, USA
}

\section{Introduction}

In the framework of our multi-frequency study of Virgo A we have performed observations of Vir A at $10.55 \mathrm{GHz}$ with the Effelsberg $100-\mathrm{m}$ telescope. Using our improved CLEAN procedure for single dish data we have increased the dynamic range to some $40 \mathrm{~dB}$.

By applying our newly developed polarization CLEANing technique we are able to diminish instrumental polarization effects. Since Faraday rotation is negligible at $\lambda 2.8 \mathrm{~cm}$ the measured linear polarization is a direct trace of the projected magnetic field in Vir A. In combination with low-frequency data obtained with the VLA it is possible to determine parameters like spectral indices, break frequencies, and spectral ages.

\section{The Radio Maps}

Sensitive maps of Virgo A have been obtained at $\lambda 2.8 \mathrm{~cm}$ using the Effelsberg 100-m telescope, including all Stokes parameters to derive the linear polarization. For spectral investigations maps have been obtained with the VLA at frequencies of $74 \mathrm{MHz}, 333 \mathrm{MHz}$, and $1.46 \mathrm{GHz}$ (Kassim et al.,1993). The lobes surrounding the central core show a roughly ' $\mathrm{S}$-shaped' structure, with two brighter components, one to the east and one to the 
southwest of the core. The perception that the extended components are radio lobes, rather than forming a diffuse halo surrounding the core (as was believed in the past), is strongly supported by the polarization data. The map of the polarized intensity exhibits the same dual structure, with an eastern and southwestern component. The polarized emission is found to be strongest at the outer edges of these components. This coincides with regions of an increased degree of polarization, with maximum values exceeding $70 \%$, which is close to the theoretical limit. This is a feature frequently seen in double-lobed radio galaxies and can be explained in terms of compression of the magnetic field as the jet material is impinging on the intracluster medium (ICM) and is deflected. Virgo A exhibits a distinct asymmetry between the southern (which is the side of the approaching jet) and the northern lobe (the counterjet side) in three respects :

- The southern region of the source shows an increased average brightness by a factor of 1.2 .

- The mean degree of polarization is higher by a factor of 1.15 on the southern side compared to the northern side of the source.

- The spectrum on the jet side is flatter than on the counterjet side.

The observed asymmetries are prevalent in powerful double radio sources with one-sided jets as described by Laing et al.(1988).

\section{Spectral investigations}

We have constructed maps, showing the distribution of the spectral index across Vir A. For this purpose three frequencies $(333 \mathrm{MHz}, 1.4 \mathrm{GHz}$, and $10.55 \mathrm{GHz}$ ) were used. The spectral indices range from $\alpha=0.4$ in the core region to $\alpha=2.2$ in the outer areas of the source $\left(S_{\nu} \sim \nu^{-\alpha}\right)$. These values are noticeably lower than the ones previously published (Andernach et al., 1979). The spectral steepening, caused by synchrotron and inverse Compton losses, can be used to calculate break frequencies $\nu_{b}$, by fitting spectral models to the data, following the method proposed by Carilli et. al. (1991). We have calculated $\nu_{b}$ for the JP (Jaffe \& Perola, 1973) model. The spectral age of the relativistic particles is computed using a relation of Alexander et al. (1987) for both radio lobes.

Eastern Lobe: $\nu_{b}=7.5 \mathrm{GHz} \quad t=36 \quad$ Myrs

Southern Lobe : $\nu_{b}=11.9 \mathrm{GHz} \quad t=29 \quad$ Myrs

\section{References}

Alexander,P., Leahy,J.P.: 1987, MNRAS 225, 1

Andernach,H.,Baker,J.R.,von Kap-herr,A.,Wielebinski.R.: 1979, $A \& A, 74,93$

Carilli,C.L.,Perley,R.A.,Dreher,J.W.,Leahy,J.P.: 1991, A p.J., 383, 554

Jaffe,W.J.,Perola,G.C.: 1973, $A \& A, \mathbf{2 6}, 423$

Kassim,N.E.,Perley,R.A.,Erickson,W.C.,Dwarakanath,K.S.: 1993, AJ, 106, 2218

Laing,R.A.: 1988, Nature, 331, 149 